\title{
Significant differences in femoral torsion values depending on the CT measurement technique
}

\author{
Peter Kaiser ${ }^{1} \cdot$ René Attal $^{1} \cdot$ Mark Kammerer $^{2} \cdot$ Michael Thauerer $^{2} \cdot$ \\ Lea Hamberger ${ }^{1} \cdot$ Raul Mayr $^{1} \cdot$ Werner Schmoelz $^{1}$
}

Received: 7 March 2016/Published online: 8 August 2016

(c) The Author(s) 2016. This article is published with open access at Springerlink.com

\begin{abstract}
Introduction This study compared the feasibility of six different CT-based measurement techniques for establishing an indication for derotational osteotomy in the cases of patellar instability or femoral fracture.

Materials and methods CT scans of 52 single human cadaver femora were measured using six different torsion measurement techniques (described by Waidelich, Murphy, and Yoshioka on transverse images and Hernandez, Jarrett, and Yoshioka on oblique images). All measurements were performed by four observers twice to assess intraobserver and interobserver agreement. The intraclass correlation coefficient (ICC), ANOVA, and Bonferroni post hoc test were used for the statistical analysis.

Results Significant differences $(P<0.001)$ between the values for femoral torsion were observed with all techniques except Yoshioka's techniques on transverse and oblique slices $(P=1.000)$ (transverse images: Waidelich $22.4^{\circ} \pm 6.8^{\circ}$, Murphy $17.5^{\circ} \pm 7.0^{\circ}$, Yoshioka $13.4^{\circ} \pm 6.9^{\circ}$; oblique images: Hernandez $11.4^{\circ} \pm 7.4^{\circ}$, Jarrett $14.9^{\circ} \pm 7.5^{\circ}$, Yoshioka oblique $13.4^{\circ} \pm 7.1^{\circ}$ ). Intraobserver and interobserver agreement showed a high level of reproducibility (ICC $0.877-0.986$; mean $0.8^{\circ}-2.9^{\circ}$ ) for all techniques, with the greatest difference being observed with Hernandez's technique $\left(11.4^{\circ} / 10^{\circ}\right)$.

Conclusions Femoral torsion values depend on the measurement technique. When derotational osteotomy is being
\end{abstract}

Werner Schmoelz

Werner.schmoelz@i-med.ac.at

1 Department of Trauma Surgery, Medical University of Innsbruck, Anichstrasse 35, 6020 Innsbruck, Austria

2 Department of Radiology, Medical University of Innsbruck, Innsbruck, Austria considered, it is essential to use different threshold values depending on the measurement technique.

Keywords Femoral · Torsion · Anteversion · Patella dislocation · Derotational osteotomy

\section{Introduction}

Femoral torsion, also known as femoral rotation or femoral version, refers to the twist between the proximal and distal parts of the femur on the transverse plane. Various imaging techniques, including radiography [9], ultrasound [4], lowdose biplanar radiography [17], computed tomography (CT) [8, 11-13, 18, 24, 25], and magnetic resonance imaging (MRI) [3, 20, 23] have been used to assess femoral torsion. With their speed, precision, and ease of use, crosssectional imaging modalities, such as CT or MRI, are regarded as the gold standard for measuring torsion in the femur.

Descriptions of various measurement techniques have been published, using transverse or oblique and single or superimposed image slices. The techniques also use different anatomical landmarks for measurement. As a result, a wide range of the standard values for femoral torsion $\left(7^{\circ}\right.$ $24.1^{\circ}$ internal torsion) has been reported in the literature [3, 5, 14, 15, 20, 21, 23-25].

The assessment of femoral torsion is important in the cases of maltorsion after a femur fracture or in the cases of lateral patellar instability, as an excessive femoral internal torsion has been described as a risk factor $[2,6,7,12,19,22]$. In the cases of recurrent patellar instability, femoral internal torsion of more than $15^{\circ}-25^{\circ}$ is considered to represent an indication for derotational femoral osteotomy $[1,2,12]$. 
The threshold range for conducting a derotational osteotomy overlaps with the range of the standard values for femoral torsion. It is, therefore, possible that patients with recurrent patellar instability in whom femoral torsion lies within the standard range might also be regarded as candidates for derotational osteotomy. As there are multiple measurement techniques, the influence of the technique on the value measured and thus on the threshold value remains unclear. Influencing factors include the use of different anatomical landmarks for measurement and high levels of intraobserver and interobserver agreement.

The purpose of the present study was, therefore, to evaluate the differences in femoral torsion values that arise due to different CT measurement techniques and the associated intraobserver and interobserver agreement. The findings may be helpful for surgeons who use CT values to establish the indication for femoral derotational osteotomy.

\section{Materials and methods}

CT scans of 26 pairs of human cadaver femora (11 female, 15 male) were used for CT measurement of femoral torsion. All femora were dissected leaving just the femoral bone itself. The donors' mean age was 73.7 years (range 51-90 years). The donors gave informed consent for medical studies to the anatomical institute during lifetime.

A LightSpeed VCT (GE Healthcare, Little Chalfont, UK) was used for scanning, with scan properties of $100 \mathrm{kV}$ and $9 \mathrm{mAs}$. The specimens were positioned with their longitudinal axis along the CT bench for scanning. The scans were all reformatted to first transverse slices with a slice distance of $2.5 \mathrm{~mm}$ and a slice thickness of $2.5 \mathrm{~mm}$; and second, oblique slices parallel to the femoral neck with a slice distance of $5 \mathrm{~mm}$ and a slice thickness of $5 \mathrm{~mm}$. Measurements were performed with the Impax EE R20 viewer (Agfa Healthcare, Mortsel, Belgium).

Femoral torsion was independently measured using six different measurement techniques by four observers (two trauma surgeons and two radiologists) for the assessment of the interobserver agreement. All the measurements were repeated after a period of $8-12$ weeks for the calculation of the intraobserver agreement. All observers were taught all measurement techniques in advance with different torsion CT images. The applied measurement techniques of femoral torsion are described in the literature and commonly used in various clinics assessed through conversation with colleagues. No anatomical analysis of the femoral torsion with a reference measurement technique, such as a goniometer or 3D surface digitizing with volume rendering, was used for validation, because the measured femoral torsion will always depend on the definition of landmarks and reference points independent of the measurement technique (goniometer, 3D surface digitizing, CT scan, etc.).

Femoral torsion was assessed by the angle between axes in the proximal and distal parts of the femur. For all the techniques, the axis in the distal part of the femur was a tangent to the posterior condyles on a single slice of a transverse image in which the condyles had their maximum expansion from anterior to posterior (Fig. 2h) [18].

Six different techniques for measuring the axis in the proximal part of the femur were used, in accordance with the following descriptions (Figs. 1, 2):

1. The technique described by Waidelich et al. on superimposed transverse slices [24]: the center of the femoral head on one transverse slice was connected to the center of an ellipse around the greater trochanter on another transverse slice that was located between the tip of the major trochanter and the minor trochanter.

2. The technique described by Murphy et al. on superimposed transverse slices [12, 18]: the center of the femoral head on one transverse slice was connected to the center of an ellipse around the base of the femoral neck on another transverse slice.

3. The technique described by Yoshioka et al. on superimposed transverse slices [25]: the center of the femoral head on one transverse slice was connected to the center of the femoral neck at its narrowest width on another transverse slice.

4. The technique described by Hernandez et al. on a single transverse slice [11]: the center of the femoral head was connected to the center of the femoral neck
Fig. 1 Level and orientation of computed tomography slice selection (left, transverse slices; right, oblique slices)
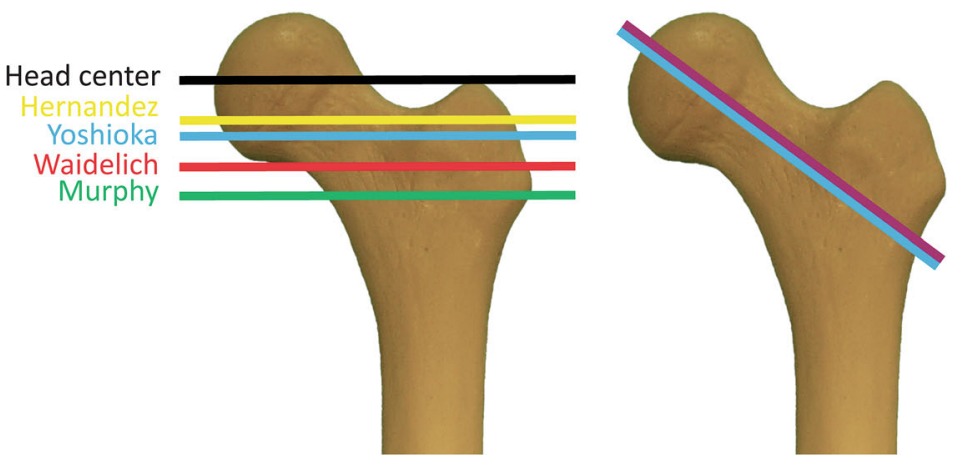

Jarrett

Yoshioka oblique 
Fig. 2 Measurement

techniques (transverse slices: a-

$\mathbf{e}, \mathbf{h}$; oblique slices: $\mathbf{f}, \mathbf{g}$ )

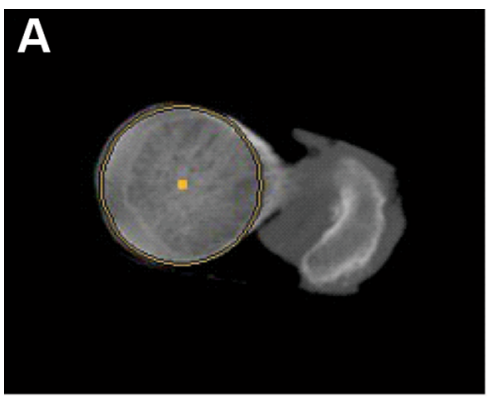

Center of femoral head

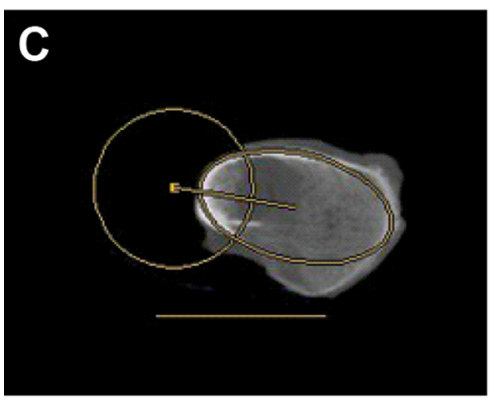

Murphy technique

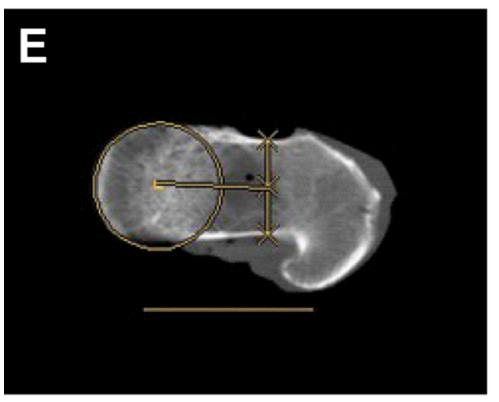

Hernandez technique

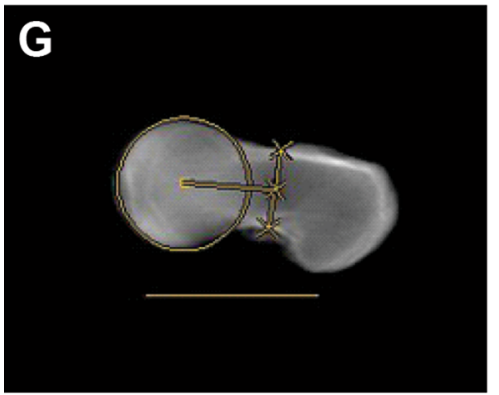

Yoshioka technique on oblique slices

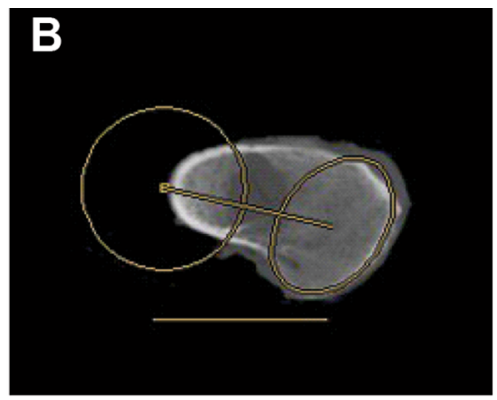

Waidelich technique

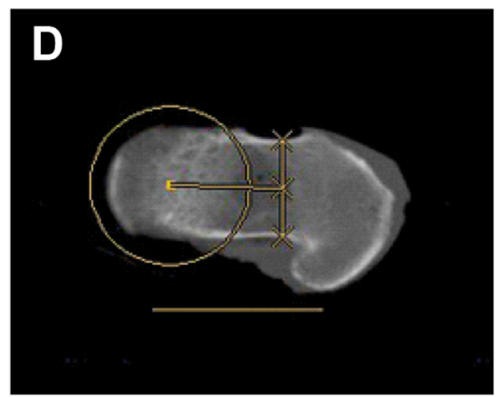

Yoshioka technique on transverse slices

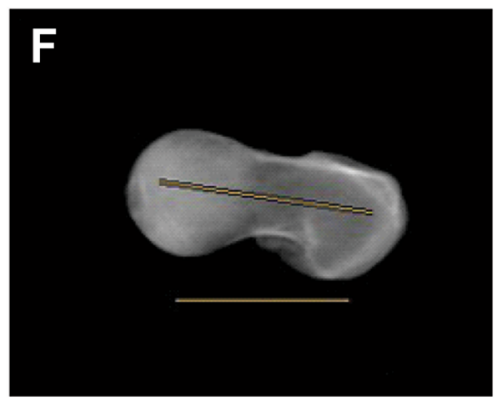

Jarrett technique

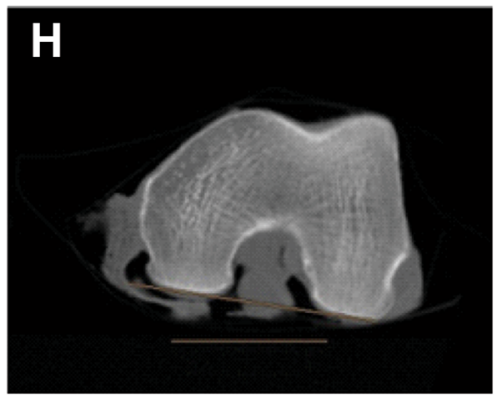

Distal axis - tangent to the posterior condyles on a single transverse slice. A slice was chosen in a location in which the femoral head, femoral neck, and major trochanter were visible.

5. The technique described by Jarrett et al. on a single oblique slice [13]: a line parallel to the femoral neck represented the proximal axis on a single oblique slice.

6. The technique described by Yoshioka et al. on superimposed oblique slices [25]: the center of the femoral head on one oblique slice was connected to the 
Fig. 3 Femoral torsion in degrees measured with different techniques $(x$ axis, measurement technique with mean and standard deviation; $y$ axis, femoral torsion in degrees (positive values $=$ antetorsion, negative values $=$ retrotorsion; Obl. = Oblique)

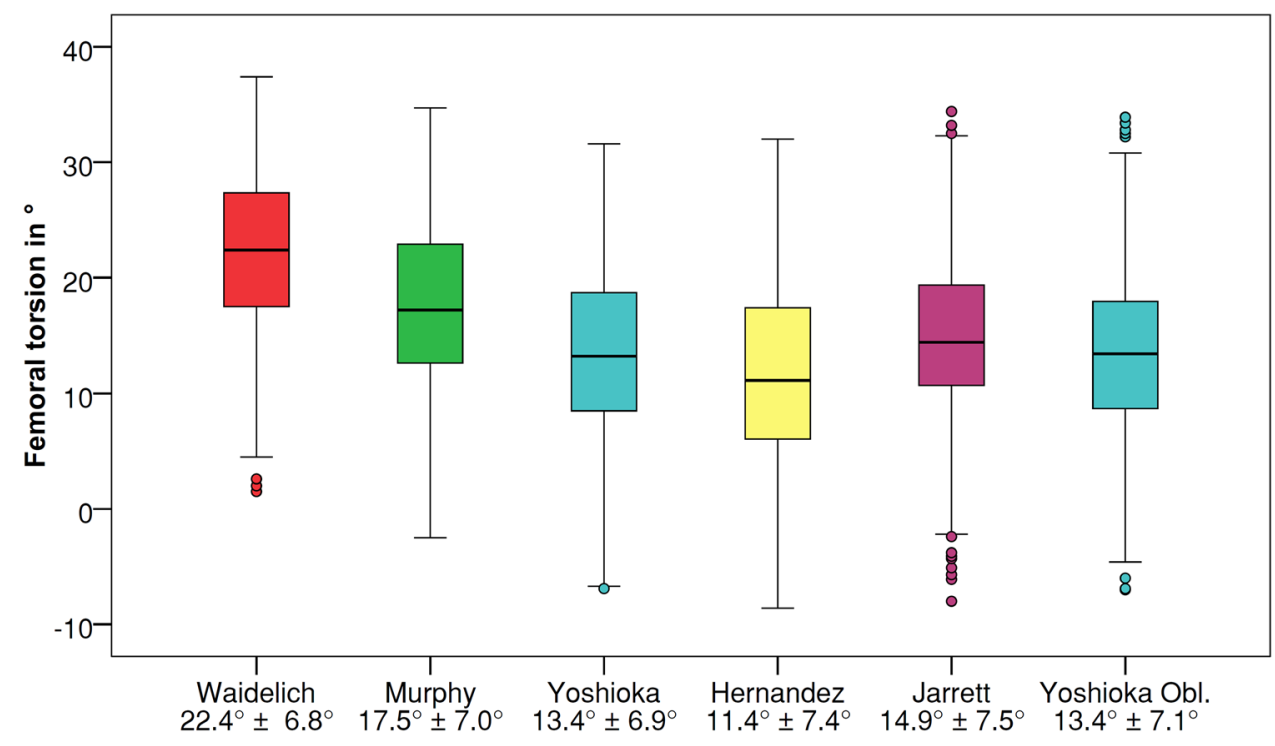

center of the femoral neck at its narrowest width on another oblique slice.

All the observers were initially instructed in these CT measurement techniques on different single-femur CT scans in advance, before the data were recorded.

The data were analyzed using IBM SPSS Statistics for Windows, Version 21.0 (Armonk, New York, USA: IBM Corporation). Descriptive values, analysis of variance (ANOVA) for repeated measurements, and the Bonferroni post hoc test were used to analyze differences between the six measurement techniques. All the measurements were included in the calculation in the comparison of the six techniques to eliminate intraobserver and interobserver agreement. Intraobserver and interobserver agreement was analyzed using the intraclass correlation coefficient (ICC) and descriptive data. The scoring system presented by Fleiss et al. [10] was used to analyze the results (ICC $>0.75$ good, $0.4-0.75$ fair, $<0.4$ poor). The significance level was set at $P<0.05$.

\section{Results}

\section{Comparison of the measurement techniques}

Significant differences were observed between pairwise comparisons of the techniques in the values measured for femoral torsion $(P<0.001)$, with the exception of Yoshioka's technique on transverse and oblique slices $(P=1.000)$. The greatest difference $\left(11^{\circ}\right)$ in the mean value for femoral torsion was found between the Waidelich and Hernandez techniques. These two techniques showed a maximum difference of up to $16^{\circ}$ in single femora. All the techniques showed similar standard deviations of approximately $7^{\circ}$ (Fig. 3).

\section{Intraobserver and interobserver agreement}

Data for intraobserver and interobserver agreement are summarized in Tables 1 and 2. The techniques all showed good intraobserver and interobserver agreement on the Fleiss et al. score [10]. The mean intraobserver and interobserver differences were small $\left(0.8^{\circ}-2.9^{\circ}\right)$. Hernandez's technique showed the largest absolute range for intraobserver and interobserver agreement $\left(11.4^{\circ}\right.$ and $13.6^{\circ}$, respectively). The distal axis at the posterior condyles showed good scores, with an ICC of 0.99 , a mean intraobserver and interobserver agreement of less than $1^{\circ}$, and maximum variance of $2.6^{\circ}$ and $3.6^{\circ}$, respectively (Tables 1,2 ).

\section{Discussion}

The most important finding of the present study was that the values measured for femoral torsion showed significant differences $(P<0.001)$ among the measurement

Table 1 Intraobserver agreement

\begin{tabular}{llll}
\hline Measurement technique & ICC & Mean & Range \\
\hline Waidelich & $0.88-0.98$ & $0.8^{\circ}-2.9^{\circ}$ & $0.0^{\circ}-8.9^{\circ}$ \\
Murphy & $0.95-0.98$ & $1.2^{\circ}-1.7^{\circ}$ & $0.0^{\circ}-6.5^{\circ}$ \\
Yoshioka & $0.94-0.97$ & $1.3^{\circ}-2.0^{\circ}$ & $0.0^{\circ}-6.6^{\circ}$ \\
Hernandez & $0.94-0.98$ & $1.2^{\circ}-2.0^{\circ}$ & $0.0^{\circ}-11.4^{\circ}$ \\
Jarrett & $0.94-0.98$ & $1.3^{\circ}-2.2^{\circ}$ & $0.0^{\circ}-5.5^{\circ}$ \\
Oblique Yoshioka & $0.94-0.99$ & $0.9^{\circ}-1.9^{\circ}$ & $0.0^{\circ}-8.1^{\circ}$ \\
Distal angle & 0.99 & $0.5^{\circ}-0.6^{\circ}$ & $0.0^{\circ}-2.6^{\circ}$ \\
\hline
\end{tabular}

ICC intraclass coefficient 
Table 2 Interobserver agreement

\begin{tabular}{llll}
\hline Measurement technique & ICC & Mean & Range \\
\hline Waidelich & $0.91-0.92$ & $2.2^{\circ}-2.6^{\circ}$ & $0.0^{\circ}-9.2^{\circ}$ \\
Murphy & 0.93 & $2.1^{\circ}$ & $0.0^{\circ}-9.4^{\circ}$ \\
Yoshioka & $0.92-0.95$ & $1.7^{\circ}-2.2^{\circ}$ & $0.0^{\circ}-10.4^{\circ}$ \\
Hernandez & $0.94-0.96$ & $1.6^{\circ}-1.9^{\circ}$ & $0.0^{\circ}-13.6^{\circ}$ \\
Jarrett & $0.92-0.94$ & $2.0^{\circ}-2.5^{\circ}$ & $0.0^{\circ}-8.9^{\circ}$ \\
Oblique Yoshioka & 0.96 & $1.5^{\circ}-1.6^{\circ}$ & $0.0^{\circ}-8.7^{\circ}$ \\
Distal angle & 0.99 & $0.7-0.8^{\circ}$ & $0.0^{\circ}-3.6^{\circ}$ \\
\hline
\end{tabular}

ICC intraclass coefficient

techniques using CT scans. Only Yoshioka's technique on transverse and oblique slices showed comparable values. The maximum differences were observed between the Waidelich and Hernandez techniques, with a maximum difference of up to $16^{\circ}$ of femoral torsion for single femora.

The mean values observed in the present study were comparable with the standard values published in the literature for the Waidelich technique (mean $20.4^{\circ}-24.1^{\circ}$ ) [21, 23, 24], Yoshioka technique $\left(13.1^{\circ}\right)$ [25], Hernandez technique $\left(12.4^{\circ}\right)$ [15], and oblique slices $\left(15.7^{\circ}-16.7^{\circ}\right)$ $[3,20,23]$. Thus, it appears to be the case that differences in the values measured for femoral torsion depend more on the measurement technique used than on the specific patient group.

Measuring femoral torsion is important when assessing risk factors for recurrent patellar instability, since increased internal femoral torsion is regarded as a factor that facilitates patellar dislocation [2, 6, 7, 12, 19, 22]. Absolute values for femoral torsion exceeding $15^{\circ}-25^{\circ}$ of internal torsion have been described as representing an indication for derotational osteotomy in patients with recurrent patellar instability $[1,2,12]$. However, these threshold values may lie within the standard range or may even represent external femoral torsion, depending on the measurement technique used (Fig. 1). Absolute threshold values for femoral torsion establishing an indication for derotational osteotomy might be pathological if the Hernandez technique is used, but might also be physiological if the Waidelich technique is used.

Fixing a femoral fracture especially by methods of closed reduction might result in excessive internal or external maltorsion. An increased internal maltorsion seems to be clinically more disabling because of an intoeing gait than an increased external maltorsion. Measurement of femoral torsion in such cases is, therefore, of major importance to assess the degree of maltorsion. The absolute value of femoral torsion needs to be reflected in regard to the correct measurement technique with its own norm values to plan the correct degree of surgical derotation and not ending up in another malttorsion.
The findings of the present study show that there is a need to use standard values dependent on the measurement technique for femoral torsion. Values for femoral torsion, therefore, have to be interpreted cautiously in relation to the reported threshold values for derotation, as the measurement technique always needs to be taken into account. Radiologic reporting should always include the technique which was used for measurement.

When femoral torsion is being assessed, it is crucial to use a technique that can be repeated with a low level of intraobserver and interobserver agreement. Femoral torsion is measured as the angle between an axis in the proximal and distal parts of the femur. The present study and the literature reports show a low level of intraobserver and interobserver agreement, with a maximum of $3.6^{\circ}$ and a mean of $1^{\circ}$ for the construction of the distal axis as a tangent to the posterior condyles [16, 18]. It, therefore, appears that the main reason for intraobserver and interobserver differences in measuring femoral torsion is the way in which the proximal axis is constructed. The results of the present study indicate good reproducibility for all of the techniques, with a low mean intraobserver and interobserver agreement of approximately $2^{\circ}$. Despite a high ICC, the technique described by Hernandez showed the greatest maximum intraobserver and interobserver differences $\left(11.4^{\circ}\right.$ and $\left.13.6^{\circ}\right)$. Similarly high values have been reported for this technique in the literature $[16,18]$. Lower values were noted with the other techniques, a finding that is in agreement with the reported results with the Waidelich [13, 23, 24], Murphy [18], and Jarrett techniques [13] for intraobserver and interobserver agreement. The reason for the higher values with Hernandez's technique might be that in some cases, the femoral head and neck cannot be visualized adequately on a single slice-especially in the cases in which there is a large femoral neck-shaft angle in the frontal plane (coxa valga) [24]—so that the slice choice for measurement may vary. Superimposed images thus appear to be better for measurement and can be recommended to determine the femoral neck axis.

One limitation of this study is that postmortem femora from elderly patients were used and the sample size was small, so that the study may not provide an adequate basis for deducing standard values for the measurement techniques. In addition, the soft-tissue mass of the thigh and a physiological position on the CT bench were not simulated.

Trauma surgeons as well as radiologists at our institution used the technique described by Waidelich for the measurement of femoral torsion already prior to this study, while the measurement technique was a topic of frequent discussion. With the present study, the currently used method was confirmed as the standard technique in our clinic, because the intra- and interobserver agreement is high and mean values are reported in the literature. 
In the conclusion, this study shows that surgeons need to be aware that threshold values for establishing an indication for derotational osteotomy and standard values for femoral torsion always need to be interpreted relative to the measurement technique used, since a pathological value measured with one technique may be physiological using the standard values from another one. With regard to intraobserver and interobserver agreement, techniques that use superimposed images or an oblique image appear to be preferable for measuring femoral torsion. Our institution uses the technique described by Waidelich because of its high intra- and interobserver agreement and the availability of norm values in the literature.

Acknowledgments The authors wish to thank the individuals who donated their bodies and tissues to advance education and research [3].

Open Access This article is distributed under the terms of the Creative Commons Attribution 4.0 International License (http://crea tivecommons.org/licenses/by/4.0/), which permits unrestricted use, distribution, and reproduction in any medium, provided you give appropriate credit to the original author(s) and the source, provide a link to the Creative Commons license, and indicate if changes were made.

\section{References}

1. Ateschrang A, Freude T, Grunwald L, Schaffler A, Stockle U, Schroter S (2014) Patella dislocation: an algorithm for diagnostic and treatment considering the rotation. Zeitschrift fur Orthopadie und Unfallchirurgie 152(1):59-67

2. Biedert RM (2008) Osteotomies. Der Orthop 37(9):872 874-876, 878-880 passim

3. Botser IB, Ozoude GC, Martin DE, Siddiqi AJ, Kuppuswami S, Domb BG (2012) Femoral anteversion in the hip: comparison of measurement by computed tomography, magnetic resonance imaging, and physical examination. Arthrosc J Arthrosc Relat Surg Off Publ Arthrosc Assoc N Am Int Arthrosc Assoc 28(5):619-627

4. Braten M, Terjesen T, Rossvoll I (1992) Femoral anteversion in normal adults. Ultrasound measurements in 50 men and 50 women. Acta Orthop Scand 63(1):29-32

5. Decker S, Suero EM, Hawi N, Muller CW, Krettek C, Citak M (2013) The physiological range of femoral antetorsion. Skelet Radiol 42(11):1501-1505

6. Dejour D, Le Coultre B (2007) Osteotomies in patello-femoral instabilities. Sports Med Arthrosc Rev 15(1):39-46

7. Dickschas J, Harrer J, Reuter B, Schwitulla J, Strecker W (2015) Torsional osteotomies of the femur. J Orthop Res Off Publ Orthop Res Soc 33(3):318-324

8. Dugdale TW, Degnan GG, Turen CH (1992) The use of computed tomographic scan to assess femoral malrotation after intramedullary nailing. A case report. Clin Orthop Relat Res 279(6):258-263

9. Dunlap K, Shands AR Jr, Hollister LC Jr, Gaul JS Jr, Streit HA (1953) A new method for determination of torsion of the femur. J Bone Jt Surg (American volume) 35(2):289-311

10. Fleiss J (1986) The design and analysis of clinical experiments. Wiley, New York

11. Hernandez RJ, Tachdjian MO, Poznanski AK, Dias LS (1981) CT determination of femoral torsion. AJR Am J Roentgenol 137(1):97-101

12. Hinterwimmer $S$, Rosenstiel $N$, Lenich $A$, Waldt $S$, Imhoff $A B$ (2012) Femoral osteotomy for patellofemoral instability. Der Unfallchirurg 115(5):410-416

13. Jarrett DY, Oliveira AM, Zou KH, Snyder BD, Kleinman PK (2010) Axial oblique CT to assess femoral anteversion. AJR Am J Roentgenol 194(5):1230-1233

14. Koerner JD, Patel NM, Yoon RS, Sirkin MS, Reilly MC, Liporace FA (2013) Femoral version of the general population: does "normal" vary by gender or ethnicity? J Orthop Trauma 27(6):308-311

15. Kuo TY, Skedros JG, Bloebaum RD (2003) Measurement of femoral anteversion by biplane radiography and computed tomography imaging: comparison with an anatomic reference. Invest Radiol 38(4):221-229

16. Liodakis E, Doxastaki I, Chu K et al (2012) Reliability of the assessment of lower limb torsion using computed tomography: analysis of five different techniques. Skelet Radiol 41(3):305-311

17. Meyrignac O, Moreno R, Baunin C et al (2015) Low-dose biplanar radiography can be used in children and adolescents to accurately assess femoral and tibial torsion and greatly reduce irradiation. Eur Radiol 25(6):1752-1760

18. Murphy SB, Simon SR, Kijewski PK, Wilkinson RH, Griscom NT (1987) Femoral anteversion. J Bone Jt Surg (American volume) 69(8):1169-1176

19. Post WR, Teitge R, Amis A (2002) Patellofemoral malalignment: looking beyond the viewbox. Clin Sports Med 21(3):521-546

20. Schneider B, Laubenberger J, Jemlich S, Groene K, Weber HM, Langer M (1997) Measurement of femoral antetorsion and tibial torsion by magnetic resonance imaging. $\mathrm{Br} \mathrm{J}$ Radiol 70(834):575-579

21. Strecker W, Keppler P, Gebhard F, Kinzl L (1997) Length and torsion of the lower limb. J Bone Jt Surg (British volume) 79(6):1019-1023

22. Teitge RA (2006) Osteotomy in the Treatment of Patellofemoral Instability. Tech Knee Surg 5(1):2-18

23. Tomczak RJ, Guenther KP, Rieber A, Mergo P, Ros PR, Brambs HJ (1997) MR imaging measurement of the femoral antetorsional angle as a new technique: comparison with $\mathrm{CT}$ in children and adults. AJR Am J Roentgenol 168(3):791-794

24. Waidelich HA, Strecker W, Schneider E (1992) Computed tomographic torsion-angle and length measurement of the lower extremity. The methods, normal values and radiation load. RoFo: Fortschritte auf dem Gebiete der Rontgenstrahlen und der Nuklearmedizin 157(3):245-251

25. Yoshioka Y, Cooke TD (1987) Femoral anteversion: assessment based on function axes. J Orthop Res Off Publ Orthop Res Soc $5(1): 86-91$ 